\title{
COMMENTS ON THE CHANGES IN AMPLITUDE OF THE CHANDLERIAN WOBBLE
}

\author{
BERNARD GUINOT
}

Bureau International de l'Heure, Paris, France

As the annual term of the polar path is not strictly equal from year to year, its separation from the Chandlerian wobble is somewhat arbitrary. However, the deviations from its mean value are small (usually 0.01 to 0.02 in semi-amplitudes) when compared to the changes in amplitude of the Chandlerian wobble.

For the interval of time 1962.0-1971.0, when the BIH coordinates of the pole in a uniform system are available, we made the separation of the two components by the method formerly applied to the Paris astrolabe results (Guinot, 1965).

Figure 1 shows the mean annual terms for two different intervals of time. They are nearly identical.

Figure 2 shows the pole path corrected for the mean annual term. A rapid change of the amplitude occurred between 1967.2 and 1967.9. However, the barycenters of the

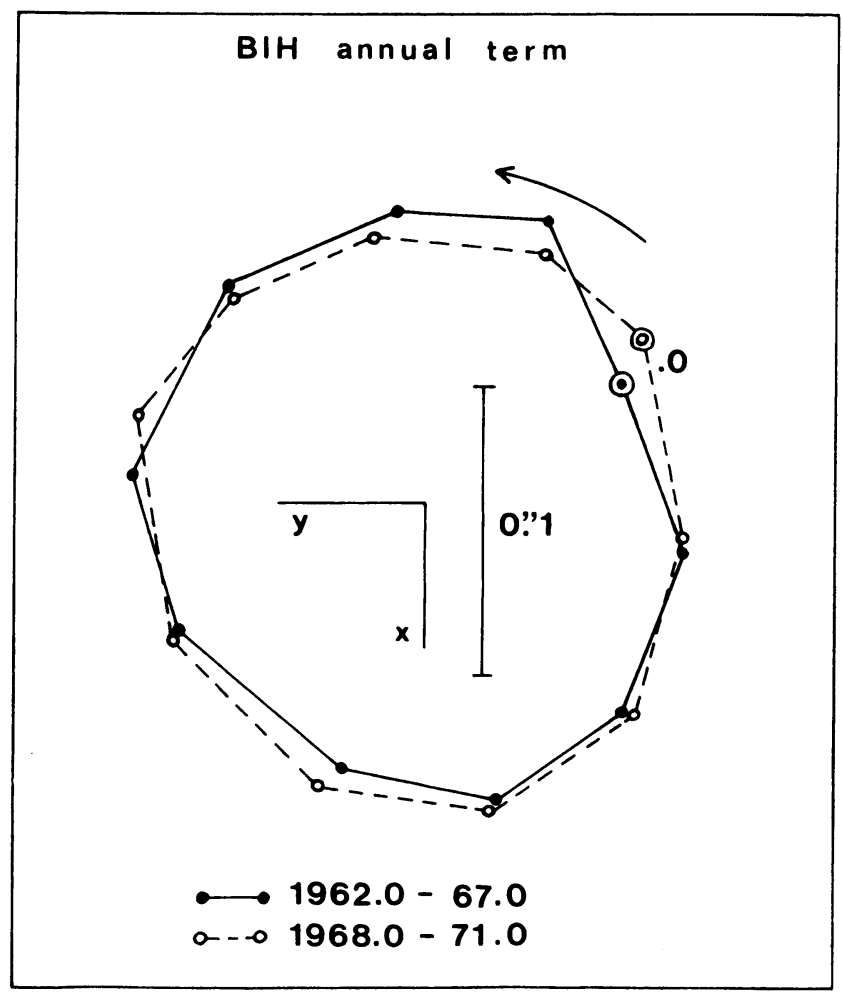

Fig. 1. The mean annual term of the BIH pole path. 


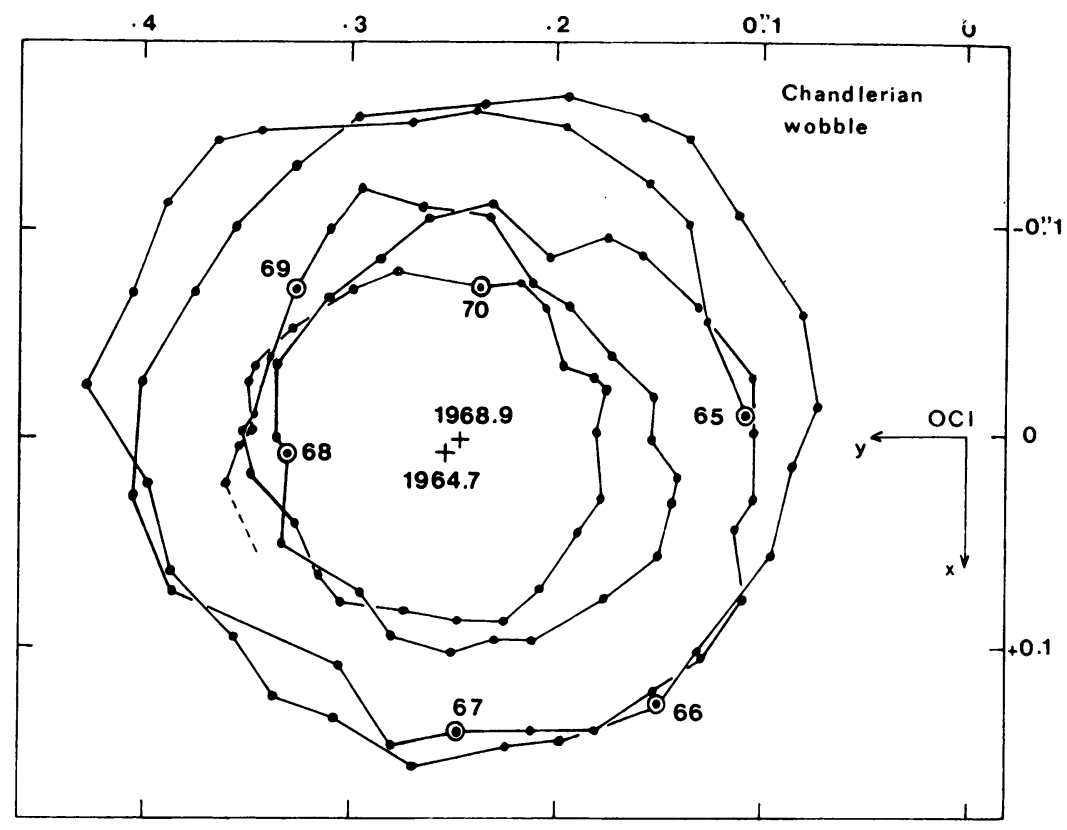

Fig. 2. Chandlerian wobble obtained by removal of the mean annual pole path.

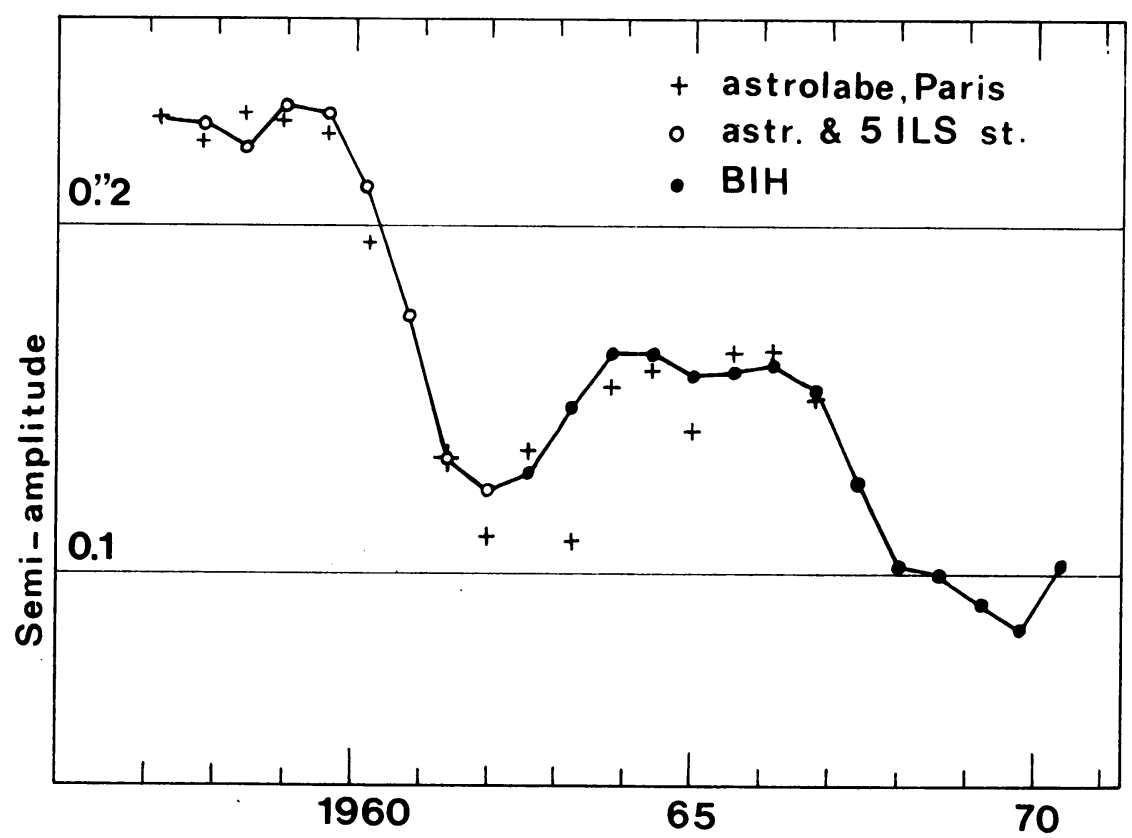

Fig. 3. Semi-amplitude of the Chandlerian wobble obtained by several combinations of instruments. 
pole path computed before and after this change almost coincide. It is also important to note that no step in phase appears. The deviation of the path from the circular form before and after the change is due to irregularities of the annual term, either real or introduced by the observations.

Figure 3 shows the variation of the amplitude of the Chandlerian wobble, obtained by adjusting sine curves of period 1.2 year over intervals of 1.2 year, after correction for annual terms, as previously explained. Another rapid change took place in 1960, with the same characteristics as the one of 1967.

Similar studies of the individual series of latitude measurement since 1900 are in progress. They indicate that such sudden changes occurred at irregular intervals (of the order of 5 years): they were not accompanied by sudden changes of the mean pole nor of the phase. There is, however, one exception, in 1926, but it may be explained by a reversal of the phase (Danjon and Guinot, 1954) or, a change in sign of the amplitude.

All these properties agree remarkably well with the hypothesis of impulsive torques developed by Runcorn (1970). They do not exclude the possibility of excitation of the Chandlerian wobble by earthquakes (Mansinha and Smylie, 1967) because the effects on the pole path of such an excitation are much smaller.

\section{References}

Danjon, A. and Guinot, B.: 1954, Compt. Rend. Acad. Sci. Paris 238, 1081.

Guinot, B.: 1965, Bull. Astron. 24, 461.

Mansinha, L. and Smylie, D. E.: 1967, J. Geophys. Res. 72, 4731.

Runcorn, S. K.: 1970, in L. Mansinha et al. (eds.), Earthquake Displacement Fields and the Rotation of the Earth, D. Reidel Publ. Comp., Dordrecht-Holland, pp. 181-187.

\section{DISCUSSION}

T. Okuda: Is there any reasonable assumption that the annual term is more stable than the Chandler term during a period of about 6-7 yr?

B. Guinot: During most of the time, the Chandlerian term and the annual term are both stable, the irregularities being small when compared to the sudden changes of the amplitude of the Chandlerian motion. Of course, when the amplitude of the Chandlerian motion changes, no determination of the annual term is possible, but after the change, the annual term assumes its previous shape. This was checked not only on the data presented here, but also on the latitudes of each ILS station.

$H$. Jeffreys: In my analysis I found the annual variation varying by a factor of about 2 . As this is usually considered to be mainly due to seasonal shifting of air masses, this suggests that these vary much from year to year; The Indian monsoon is a byproduct of these, and it does in fact vary much from year to year.

B. Guinot: I could not find such large variations in the annual motion when it can be determined. 